\title{
Ultrasound in pediatric orbital cellulitis
}

\author{
Ana Falticeanu', Pierre Tritschler ${ }^{2}$, Dana Dumitriu ${ }^{3}$
}

${ }^{1}$ Université catholique de Louvain, CHU UCL Namur, Department of Radiology Yvoir, ${ }^{2}$ Centre Hospitalier Régional De Namur, Department of Medical Imaging, ${ }^{3}$ Cliniques Universitaires Saint Luc, Brussels, Pediatric Radiology, Woluwe-Saint-Lambert, Belgium

\section{To the Editor,}

A 7-year-old presented to the emergency room with fever and right eyelid swelling. Blood tests showed increased C-reactive protein and a high white blood cell count. Ultrasound revealed signs of preseptal cellulitis and antibiotic treatment was started immediately. Two days later, swelling of the eyelid increased and painful eye movements appeared. The control ultrasound demonstrated persistence of preseptal cellulitis and displacement of the medial rectus muscle by swollen hyperechoic intraorbital fat (fig 1), suggesting retro-septal extension. The patient was admitted to the paediatric ward with good clinical evolution.

The inflammation of the soft tissues of the eye anterior to the orbital septum is referred to as preseptal cellulitis. Extension into the eye socket behind the orbital septum is referred to as orbital cellulitis (or retro-orbital/ retro-septal cellulitis) - this generic term sometimes leads to confusion regarding the definitions of periorbital (preseptal) versus orbital (retro-septal) cellulitis.

Orbital cellulitis is an acute local spread of sinus infection in more than $90 \%$ of cases in children [1]; common infectious agents are Streptococcus aureus, Streptococcus pneumoniae and Haemophilus influenzae [2]. Common clinical manifestations include swelling of the orbital soft tissues, proptosis, painful eye movement, ophthalmoplegia and fever. The diagnosis is clinical [3].

Received 04.02.2020 Accepted 25.03.2020

Med Ultrason

2020, Vol. 22, No 2, 253-254, DOI: 10.11152/mu-2438,

Corresponding author: Ana Falticeanu

Université catholique de Louvain,

CHU UCL Namur, Department of Radiology

1 Avenue Dr G Thérasse, 5530, Yvoir, Belgium

E-mail: ana.falticeanu@uclouvain.be

Phone: $+32(0) 81423533$

Fax: +32 (0)81423505
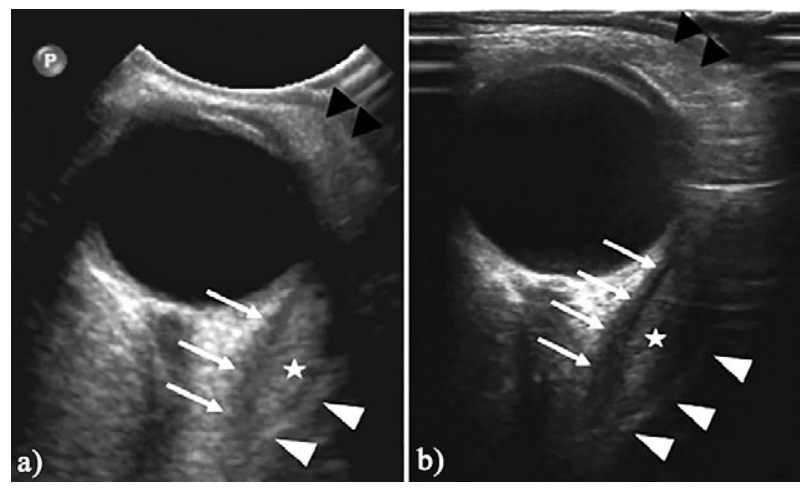

Fig 1. Axial plane of the right orbit (a: $8 \mathrm{MHz}$ probe, b: $12 \mathrm{MHz}$ probe) shows lateral displacement of the medial rectus muscle (white arrows) by the intraorbital fat (white star) with hyperechoic swelling found between the medial rectus muscle and medial orbital wall (white arrowheads); swelling of soft tissues of the eyelid is present (black arrowheads).

Ultrasound is a fast, non-invasive, non-irradiating tool. A high-frequency transducer should be used, with the eyelid closed. Pre-septal cellulitis is defined by a hyperechoic infiltration of the soft tissues of the eyelid accompanied by Doppler hyperemia. In post-septal (orbital) cellulitis hyperechoic swelling of the intra-orbital fat and medial displacement of the medial rectus muscle can be seen. More than $50 \%$ of cases are complicated by a subperiosteal abscess: an anechoic mass bulging from the medial orbital wall $[2,4,5]$. Intracranial complications are estimated at around $10 \%[6,7]$. Patients with neurological deterioration, an increase in the size of the subperiosteal abscess or lack of improvement with antibiotic treatment should undergo brain CT or MRI $[7,8]$.

In conclusion: orbital cellulitis is a potentially serious complication of acute sinusitis. Ultrasonography should be considered first when faced with an orbital cellulitis suspicion in paediatric patients as it allows a reliable differentiation between pre- and post-septal extension. 


\section{References}

1. Oxford LE, McClay J. Complications of Acute Sinusitis in Children. Otolaryngol Neck Surg 2005;133:32-37.

2. Derr C, Shah A. Bedside ultrasound in the diagnosis of orbital cellulitis and orbital abscess. Emerg Radiol 2012;19:265-267.

3. Wald ER, Applegate KE, Bordley C, et al. Clinical Practice Guideline for the Diagnosis and Management of Acute Bacterial Sinusitis in Children Aged 1 to 18 Years. Pediatrics 2013;132:e262-e280.

4. Nageswaran S, Woods CR, Benjamin DK Jr, Givner LB, Shetty AK. Orbital Cellulitis in Children. Pediatr Infect Dis J 2006;25:695-699.
5. Mouriaux F, Rysanek B, Babin E, Cattoir V. Les cellulites orbitaires. J Fr Ophtalmol 2012;35:52-57.

6. Sharma PK, Saikia B, Sharma R. Orbitocranial Complications of Acute Sinusitis in Children. J Emerg Med 2014;47:282-285.

7. Herrmann BW, Forsen JW Jr. Simultaneous intracranial and orbital complications of acute rhinosinusitis in children. Int J Pediatr Otorhinolaryngol 2004;68:619625 .

8. Goytia VK, Giannoni CM, Edwards MS. Intraorbital and Intracranial Extension of Sinusitis: Comparative Morbidity. J Pediatr 2011;158:486-491.

\title{
Ultrasound-guided caudal epidural injection in three simple steps
}

\author{
Mustafa Turgut Yildizgoren
}

Fizikon Medical Center, Department of Physical Medicine and Rehabilitation, Konya, Turkey

\section{To the Editor,}

Caudal epidural injection can be helpful in the symptomatic treatment of radicular pain due to spinal stenosis or lumbar disc herniations [1]. Successful caudal injection relies on the accurate placement of a needle into the epidural space through the sacral hiatus. Incorrect needle placement occurs at a rate of up to $30 \%$ patients when performed using the blind technique [2]. It can be difficult to identify the sacral hiatus by the palpation of anatomical landmarks in adults. We suggest an ultrasound-guided approach for caudal epidural injection in three steps.

The patient lies in a prone position and the lumbosacral area is prepared aseptically for injection. The first two steps are performed by using the transverse ultrasonographic view and the third step is performed by using the longitudinal ultrasonographic view (Supplementary video, on the journal site):

Step 1. The transducer is first placed transversely (axial) proximal of the anus at the midline, and where the sacral bone is seen (fig 1a);

Received 22.02.2020 Accepted 28.03.2020

Med Ultrason

2020, Vol. 22, No 2, 254-255, DOI: 10.11152/mu-2466,

Corresponding author: Mustafa Turgut Yildizgoren, MD

Fizikon Medical Center, Department of Physical Medicine and Rehabilitation, Konya, Turkey

Phone: 90 332.353.6900; Fax: 90 332.353.6900

E-mail: ftr.mustafaturgut@hotmail.com
Step 2. The transducer is moved cranially until the two sacral cornua, base of sacrum, sacrococcygeal ligament and sacral hiatus appear (fig 1b). The two sacral cornua and base of sacrum appear as two hyperechoic structures. The sacral hiatus appears in the hypoechoic region between the hyperechoic structures;

Step 3. At this level, the transducer is rotated $90^{\circ}$ to rest between the two cornua and to obtain the longitudinal sonographic view of the sacral hiatus. Sacrum, sacrococcygeal ligament and sacral hiatus are identified (fig 1c). Under the longitudinal view, the needle is inserted using the "in-plane" technique.Loss of resistance is usually felt after penetrating the sacrococcygeal ligament. After the "pop" feeling of ligament penetration, the needle is advanced further into the sacral canal, but cannot be visualized beyond the apex of the sacral hiatus.

Ultrasound-guided caudal injection technique has been shown to have very high rates of accuracy in correct needle placement into the sacral canal for subsequent epidural injections.

\section{References}

1. Chen CP, Lew HL, Tsai WC, Hung YT, Hsu CC. Ultrasound-guided injection techniques for the low back and hip joint. Am J Phys Med Rehabil 2011;90:860-867.

2. Barham G, Hilton A. Caudal epidurals: the accuracy of blind needle placement and the value of a confirmatory epidurogram. Eur Spine J 2010;19:1479-1483. 


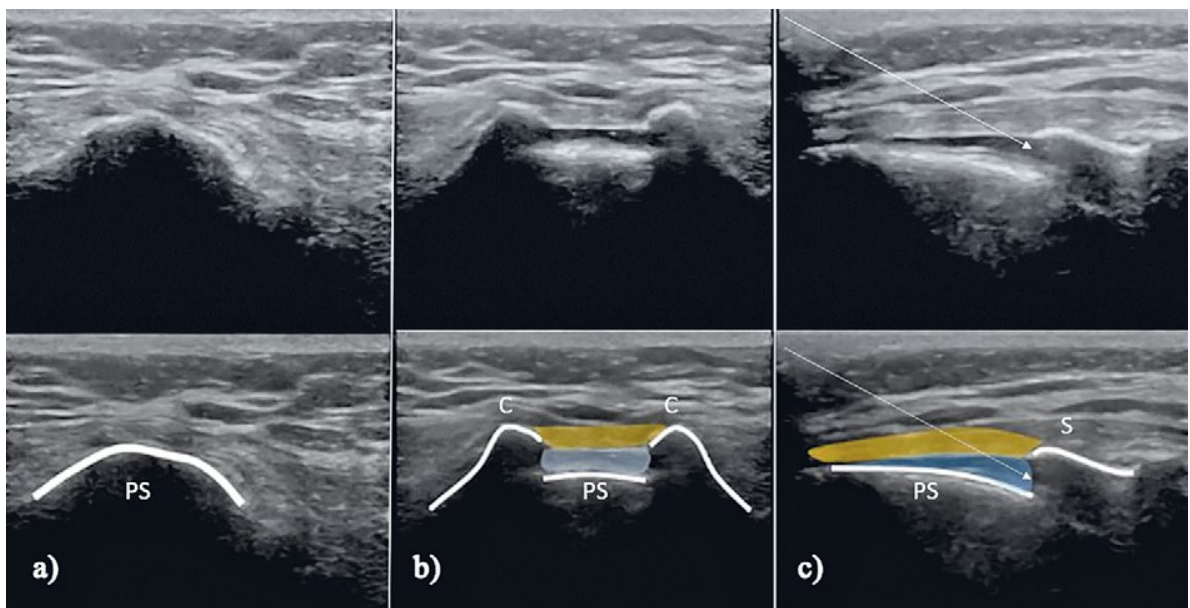

Fig 1. Ultrasound-guided caudal epidural injection: a) Axial image showing the sacral bone (curved line) proximal to the anus in the midline; b) Axial image showing the two sacral cornua, sacrococcygeal ligament and sacral hiatus. The two sacral cornua appear as inverted U-shaped structures with acoustic shadowing, and a frog's eye sign, with the posterior surface of the sacrum. The sacrococcygeal ligament can be seen between the two sacral cornua (yellow area) and underlying sacral bone, with the sacral hiatus located between these two structures (blue area); c) Longitudinal image showing the needle position (arrow). For the in-plane approach through thesacral hiatus, a 21-gauge, echogenic needle can be used. $\mathrm{S}=$ sacral bone; $\mathrm{C}=$ sacral cornua; $\mathrm{PS}=$ posterior surface of sacrum

\title{
A rare case of pericardial lymphoma in a heart transplantation recipient
}

\author{
Chun Wu, Li Zhang, Yuman Li, Mingxing Xie
}

Department of Ultrasound, Union Hospital, Tongji Medical College, Huazhong University of Science and Technology, Hubei Province Key Laboratory of Molecular Imaging, Wuhan, China

\section{To the Editor,}

A 61-year-old male patient with severe coronary artery disease received a heart transplant (HT) in December 2016. The patient underwent an uneventful perioperative course and remained on standard immunosuppression

Received 24.02.2020 Accepted 28.03.2020

Med Ultrason

2020, Vol. 22, No 2, 255-256, DOI: 10.11152/mu-2467,

Corresponding author: Mingxing Xie

Department of Ultrasound, Union Hospital, Tongji Medical College, Huazhong University of Science and Technology,

1277 Jiefang Ave, Wuhan 430022, China

Phone: 86-27-85726430

Fax: 86-27-85726172

Email: Xiemx@hust.edu.cn procedure. Fourteen months after HT, he was admitted due to severe lower-extremity edema and fainting. Laboratory investigations showed the elevated levels of EBVDNA $(2.0 \times 104$ copies). Transthoracic echocardiogram (TTE) revealed a hypoechoic mass within the pericardium $(9.6 \times 2.5 \mathrm{~cm})$ surrounding the right ventricular $(\mathrm{RV})$ free wall (fig 1a). Contrast-enhanced ultrasonography (CEUS) demonstrated the high enhancement of contrast agent in the pericardial mass (fig 1b). Positron emission tomography showed intense metabolic activity within the mass (fig 1c). Biopsy of the pericardial mass was performed for a definitive diagnosis and histopathologic examination revealed monomorphic diffuse large-B cell lymphoma (DLBCL) (fig 1d).

Subsequently, the immunosuppressive regimen of the patient was changed to dose reduced tacrolimus $(0.5 \mathrm{mg}$ 

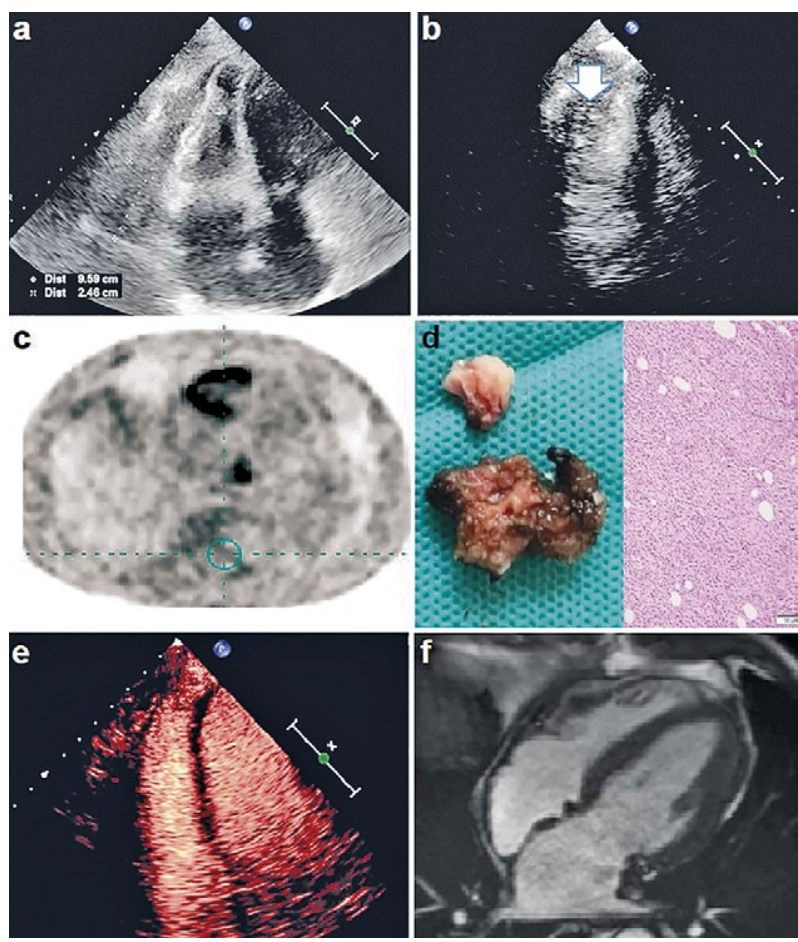

Fig 1. Transthoracic echocardiogram showing a mass within the pericardium surrounding the right ventricular free wall (a); contrast-enhanced echocardiography demonstrating the high enhancement of contrast agent in the pericardial mass (b); positron emission tomography revealing intense metabolic activity within the pericardial (c); histopathologic examination revealing diffuse large-B cell lymphoma (d); after 1-year treatment, no obvious mass is observed on contrast-enhanced echocardiography (e); Cardiac magnetic resonance imaging showing no mass within the pericardium (f). every other day), mycophenolate mofetil (500 mg once daily) and prednisolone (10 mg once daily). After 1-year treatment, no obvious pericardial mass was observed by TTE and CEUS (fig 1e). Furthermore, cardiac magnetic resonance imaging confirmed no mass within the pericardium (fig 1f). Finally, the administration of immunosuppression was adjusted to tacrolimus $(1.5 \mathrm{mg}$ once daily), mycophenolate mofetil (500 mg twice daily) and prednisolone (10 mg once daily). At the $20-$ months follow-up, the patient has been doing well without evidence of tumor recurrence.

Post-HT malignant diseases remain a major threat in HT recipients because of the high mortality and morbidity [1]. To the best of our knowledge, this may be the first case of DLBCL involving the pericardium in HT recipients. Our case confirmed that if the lesion was localized, RI was enough to induce complete remission as experienced by patients in the Reshef et al study [2]. Our case emphasizes that early diagnosis and early intervention with RI is necessary for good outcome of this rare pericardial lymphoma after HT.

\section{References}

1. Oechslin E, Kiowski W, Schneider J, Follath F, Turina M, Gallino A. Pretransplant malignancy in candidates and posttransplant malignancy in recipients of cardiac transplantation. Ann Oncol1996;7:1059-1063.

2. Reshef R, Vardhanabhuti S, Luskin MR, et al. Reduction of immunosuppression as initial therapy for posttransplantation lymphoproliferative disorder. Am J Transplant 2011;11:336-347. 